\title{
Coexistence of Temporo-Occipital Polymicrogyria with Choroidal Fissure Cyst in a Case of Focal Onset Seizures
}

\author{
$\begin{array}{llll}\text { M. Kiran } & \text { A. Karthik } & \text { L. V. Lekshmi } & \text { K. Nagarajan }\end{array}$ \\ ${ }^{1}$ Department of Radio-Diagnosis, Jawaharlal Institute of \\ Postgraduate Medical Education and Research (JIPMER), \\ Pondicherry, India \\ Address for correspondence K. Nagarajan, Department of \\ Radio-Diagnosis, Jawaharlal Institute of Postgraduate Medical \\ Education and Research (JIPMER), Pondicherry 600506, India \\ (e-mail: Iknagarajan1@gmail.com).
}

Int J Epilepsy 2018;5:104-106

\begin{abstract}
Keywords

- polymicrogyria

- choroidal fissure cyst

- seizures

$-M R I$

Focal-onset seizures can be caused by underlying brain lesions including focal lesions such as granulomas, low-grade neoplasms, vascular lesions, or neuronal migration disorders. Polymicrogyria is a congenital abnormality of cortical formation occurring during embryonic life. Choroidal fissure cysts are either arachnoid or neuroepithelial cysts arising at the choroidal fissure, and mostly they are incidental findings having no significant clinical implications. Coexistence of both of these can lead to dilemma in the management decisions. We present a case of focal-onset seizures with an unreported coexistence of polymicrogyria with choroidal fissure cyst.
\end{abstract}

\section{Introduction}

Focal lesions such as granulomas, low-grade neoplasms, vascular lesions, or neuronal migration disorders can be causative factors in adult-onset seizures. ${ }^{1}$ Polymicrogyria (PMG) is an abnormality of fissures and sulcation due to various insults occurring during cortical development in embryonic life. ${ }^{2}$ It may manifest as seizures and usually diagnosed early in life with the help of magnetic resonance imaging (MRI). Choroid fissure cysts (CFCs) are mostly incidental findings in brain MRI of general population, but may be of significance in those presenting with seizures as previous reports have mentioned their association with complex partial seizures. ${ }^{3-5}$

\section{Case Report}

A 20-year-old male patient presented with recurrent headaches and seizures since 6 years of age. He was prescribed an antiepileptic drug by a practitioner near his home but continued to have seizures. His seizures were preceded by an aura of vertigo with visual disturbances in both eyes followed by eye deviation, neck turning to left side, oral automatisms, impaired awareness of the episode, and bimanual posturing. But there was no loss of posture or bilateral spread during event. There was history of nocturnal events and sudden on-and-off events. No post ictal phenomenon or memory was present for the event. There was history of unresponsiveness and behavioral arrest during the event. The last episode was a day prior to presentation to the hospital. His intellectual and behavioral features were normal. Systemic examination was normal. Electroencephalogram revealed abnormal interictal epileptiform discharges in right posterior leads, with generalized slow wave discharges. He was diagnosed to have focal nonmotor onset sensory (visual) seizures with impaired awareness and was referred for imaging. Plain computed tomography brain revealed fluid-density lesion lateral to midbrain in the right choroid fissure region. MRI of the brain (plain and contrast) was performed. A well-defined lobulated cerebrospinal fluid (CSF) intensity lesion ( - Fig. 1) was seen medial to mediotemporal lobe on right side. There was a mass effect in the form of compression with associated partial inversion of hippocampus. Mild prominence of the temporal horn of ipsilateral lateral ventricle was noted with lateral displacement of choroid plexus. There was no diffusion restriction or contrast enhancement suggesting CFC. Multiple small gyri and sulci are noted in ipsilateral temporo-occipital lobe with normal signal as compared with rest of the brain parenchyma suggesting PMG (-Fig. 2). received

December 12, 2018 accepted after revision January 23, 2019 published online March 19, 2019
DOI https://doi.org/

10.1055/s-0039-1683836

ISSN 2213-6320.
Copyright @2018 Indian Epilepsy Society
License terms

(요 (1) $\Theta \circledast$ 


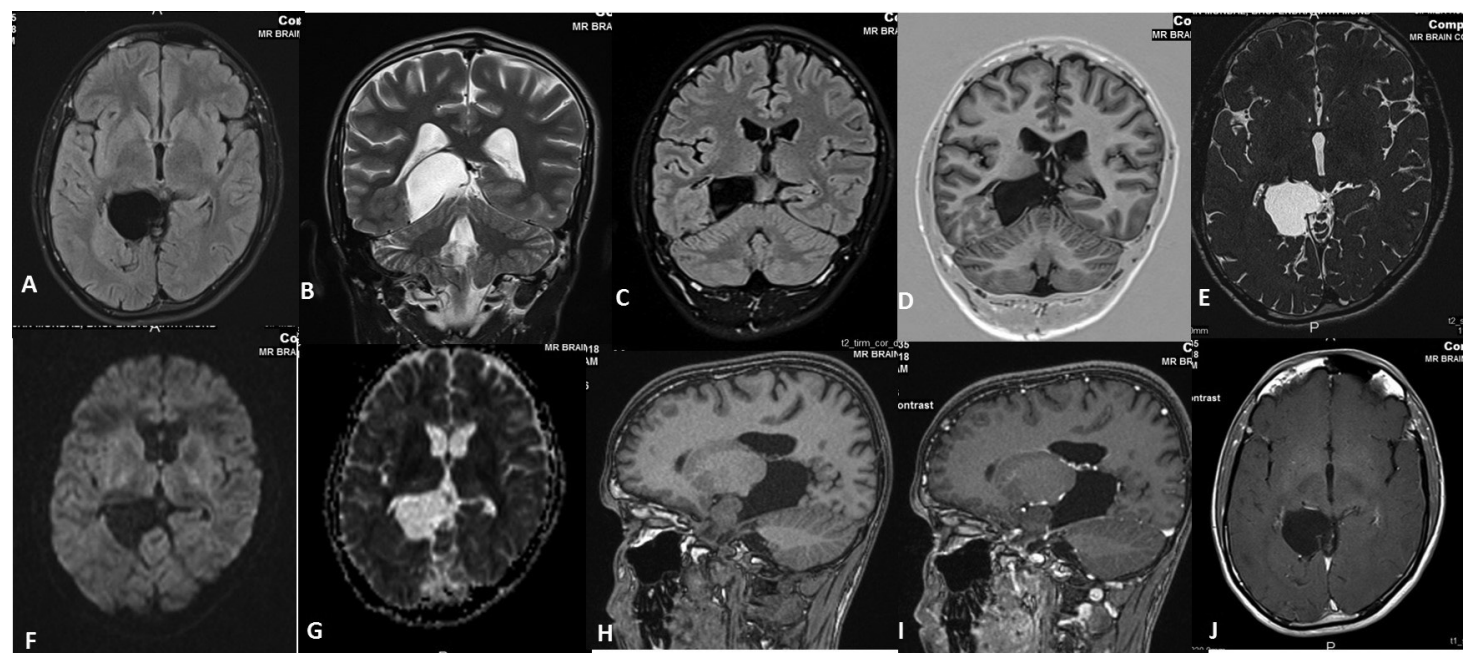

Fig. 1 Magnetic resonance imaging (MRI) fluid-attenuated inversion recovery (FLAIR) axial (A), T2 coronal (B), oblique T2 and T1 inversion recovery (IR) (C and D) coronal, heavily T2-weighted axial 3D-SPACE (sampling perfection with application-optimized contrast using different flip angle evolution) (E), diffusion-weighted image (DWI) (F), ADC (apparent diffusion coefficient) (G), T1 sagittal (H), post-contrast T1 sagittal (I), and axial (J) images showing cerebrospinal fluid (CSF)-like fluid-density lesion (arrows) in the region of right choroidal fissure without any diffusion restriction.

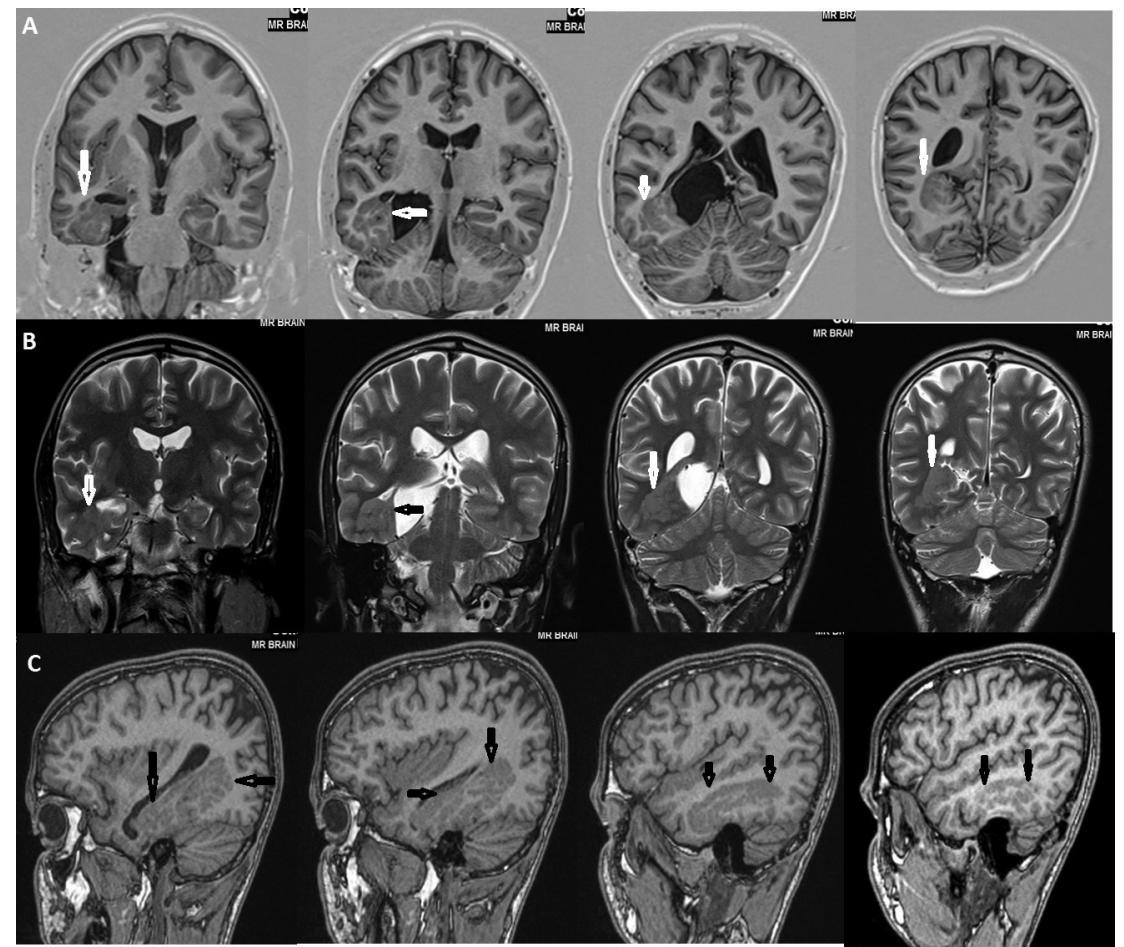

Fig. 2 Magnetic resonance imaging (MRI) T1 inversion recovery (IR) oblique coronal (A), T2 coronal (B), and T1 sagittal (C) showing multiple small gyri and altered gray-white pattern of polymicrogyria.

\section{Discussion}

Routine neuroimaging is recommended for all patients having seizures, particularly those in adult-onset and with focal type of seizures, at least once, usually before starting antiepileptic therapy. The likelihood of underlying lesions is more in those with refractory seizures and may benefit from MR neuroimaging to delineate lesion for management and if necessary, by surgical planning. ${ }^{6}$ PMG is a neuronal developmental disorder characterized by presence of multiple small partly fused gyri and sulci, and irregularly appearing cortical surface as suggested by its name. It is due to abnormal cortical formation resulting from disturbance in cortical development late in the neuronal migration stage or early in the cortical organization stage. ${ }^{2}$ Causes are multifactorial ranging from prenatal infection, ischemia, or exposure to toxins, to chromosomal abnormalities. The common patterns of PMG are perisylvian (61\%) with parasagittal parieto-occipital accounting for $3 \%$, and there is association with periventricular gray matter heterotopias in $11 \%$. In perisylvian PMG, 
$85 \%$ are bilateral and are symmetrical. ${ }^{7}$ The median age at presentation noted in the former study is 4 months. Nearly more than one-third (38\%) of cases were diagnosed in either antenatal or neonatal period. Seizures are the most common clinical sequelae of PMG with approximately $80 \%$ of patients eventually developing seizures and majority within the first 5 years. $^{7}$ Other symptoms can be encountered depending on the area of the brain involved. Bilateral abnormalities may show various syndromic associations. On MRI, the diagnostic criteria for PMG include unusually thickened and over-folded gray matter, cortical surface irregularity, and "stippling" or irregularity at gray-white matter interface. ${ }^{7}$ It appears as multiple cortical convolutions and shallow sulci with thickened or normal cortex. An anomalous vein may occasionally be seen in the region of PMG. MRI is the imaging technique of choice for diagnosing PMG. ${ }^{8}$ The management of PMG constitutes antiepileptic drugs and timely follow-up.

Choroid fissure cysts are CSF-like fluid-containing benign cysts that may be congenital in origin. The CFC can be of arachnoid or neuroepithelial origin, differentiated only at pathology. ${ }^{9}$ However, most are not confirmed with histopathology as they do not require surgery. There are reports of association of CFC with seizures, attention-deficit hyperactivity disorder, migraine, and narcolepsy. ${ }^{3-5,10,11}$ Choroid fissure is a C-shaped cleft formed by invagination of choroid plexus into the ventricle between the thalamus and fornix during the embryonal life. Tela choroidea and tela fimbria are, respectively, the attachments of villous choroid plexus to thalamus and fornix at the level of temporal horn of lateral ventricles. The fimbria and the choroid plexus are a barrier between choroidal fissure and temporal horn of lateral ventricle. ${ }^{12,13}$ This landmark is important, as in the case of arachnoid origin, choroid plexus is displaced laterally from choroidal fissure, and medially displaced if it is of neuroepithelial (ventricular) origin. At MRI CFCs are typically well-defined unilocular, smoothly marginated CSF-like fluid-containing cysts exerting mass effect on the adjacent structures. They do not show contrast enhancement or adjacent edema or gliosis. CFCs are found incidentally, and the treatment is conservative with interval follow-up, if necessary. ${ }^{9}$ If the lesion is large and symptomatic, then surgical treatment includes cyst fenestration or cystoperitoneal shunting. ${ }^{13}$

Usually, the patients of cortical malformations clinically manifest at a much younger age. Our case report emphasizes the importance of looking for more subtle abnormalities such as PMG even in the presence of an obvious lesion of CFC while evaluating an adult patient presenting with seizure for the first time without any history of trauma or headache. Subtle changes of PMG can be missed when associated with a cystic lesion as the cyst itself can have cortex-deforming mass effect as noted in our case. There have been no reported cases showing coexistence of CFC and PMG. The importance of this coexistence lies in treatment decisions as both conditions are associated with seizures. Seizures if associated with only CFCs may resolve with decompression or fenestration of the cyst or shunting. The presence of associated PMG may warrant long-term treatment with antiepileptic drugs if surgical resection is not feasible. In our patient, the patient's seizures were under control with an antiepileptic drug. This case highlights the dilemma as and when if seizures become refractory in such a patient with dual pathology. The neurosurgery opinion is to perform a decompression or excision of the cyst first, if seizures become refractory, and then to look for control of seizures with medical therapy. The patient is being followed up on an outpatient basis.

\section{Conflict of Interest}

None declared.

\section{References}

1 Craven IJ, Griffiths PD, Bhattacharyya D, et al. 3.0 T MRI of 2000 consecutive patients with localisation-related epilepsy. Br J Radiol 2012;85(1017):1236-1242

2 Jansen A, Andermann E. Genetics of the polymicrogyria syndromes. J Med Genet 2005;42(5):369-378

3 Morioka T, Nishio S, Suzuki S, Fukui M, Nishiyama T. Choroidal fissure cyst in the temporal horn associated with complex partial seizure. Clin Neurol Neurosurg 1994;96(2):164-167

4 Isolan GR, Bianchin MM, Torres CM, Bragatti JA, Assman JB, Falcetta FS. Temporal choroidal fissure cyst and temporal lobe epilepsy: report of two cases. J Epilepsy Clin Neurophysiol 2010;16(4):167-169

5 Tamayo JA, Ojea T, Hervás M, León A, Fernández O. [Neuroepithelial cyst of the choroid sulcus as a probable cause of symptomatic focal epilepsy]. Rev Neurol 1997;25(148):2070-2071

6 Bronen RA, Fulbright RK, Spencer DD, et al. Refractory epilepsy: comparison of MR imaging, CT, and histopathologic findings in 117 patients. Radiology 1996;201(1):97-105

7 Leventer RJ, Jansen A, Pilz DT, et al. Clinical and imaging heterogeneity of polymicrogyria: a study of 328 patients. Brain 2010;133(Pt 5):1415-1427

8 Mavili E, Coskun A, Per H, Donmez H, Kumandas S, Yikilmaz A. Polymicrogyria: correlation of magnetic resonance imaging and clinical findings. Childs Nerv Syst 2012;28(6):905-909

9 de Jong L, Thewissen L, van Loon J, Van Calenbergh F. Choroidal fissure cerebrospinal fluid-containing cysts: case series, anatomical consideration, and review of the literature. World Neurosurg 2011;75(5-6):704-708

10 Wang PJ, Lin HC, Liu HM, Tseng CL, Shen YZ. Intracranial arachnoid cysts in children: related signs and associated anomalies. Pediatr Neurol 1998;19(2):100-104

11 Rosich-Pla A, Smith BH, Sil R. Congenital arachnoid cyst with unusual clinical, radiological, and pathological findings. Ann Neurol 1977;2(5):443-446

12 Holodny AI, George AE, Golomb J, de Leon MJ, Kalnin AJ. The perihippocampal fissures: normal anatomy and disease states. Radiographics 1998;18(3):653-665

13 Zemmoura I, Velut S, François P. The choroidal fissure: anatomy and surgical implications. Adv Tech Stand Neurosurg 2012;38:97-113 